\title{
Complex Analysis Electronic Course in Nomotex DLS
}

\author{
Yury I. Dimitrienko ${ }^{1}$, Oleg D. Algazin ${ }^{1}$, Oksana V. Skudneva ${ }^{1}$, Julia $V$. Ozhgibisova $^{1}$, Raisa \\ $K$. Alesina ${ }^{1}$, and Alexander $V$. Alesin ${ }^{1 *}$ \\ ${ }^{1}$ Bauman Moscow State Technical University, 2nd Baumanskaya str., 5/1, 105005, Moscow, Russia
}

\begin{abstract}
Information about the electronic course "Complex Analysis" created in the Digital Learning System NOMOTEX is presented. The analysis of the results of experimental teaching of students of the Bauman Moscow State Technical University on this course.
\end{abstract}

\section{Introduction}

The section DLS NOMOTEX «Complex analysis» was developed in 2018-2020 as a module for teaching the subject «Theory of functions of a complex variable» and «Operational calculus» to second-year students of faculties «Power Engineering» all specialties, and «Fundamental Sciences», the department «Calculus Mathematics and the Mathematical Physics». Subsequently the section was also included in course «Multiple integral, Series and theory of functions of a complex variable» for students of the Aerospace Faculty.

\section{E-learning «Complex analysis»}

The e-learning «Complex analysis» contains the following sections (called 'Chapter' [1-3]): «Complex variable function concept», «Sequences and Series in Complex Analysis», «Continuity and differentiability of a function of a complex variable», "Conformal mappings», «Integrals of a complex variable function», "Integral Cauchy Formula», «Expansion of analytic functions in Taylor and Laurent series», «Equilibrium points and residues».

Each chapter of the lecture block contains several paragraphs (Fig. 1), which are divided into quanta. Micro-control tests (MCT) are based on lecture materials - small test to control student's knowledge and attendance. MCT is carried out at lecture for 10 minutes. The points for the implementation of the MCT are displayed in the personal account of students and the teacher. Missing lectures and lack of points for MCT threatens with inadmissibility to the exam or inability to get credit, depending on the form of knowledge control for the corresponding program.

\footnotetext{
* Corresponding author: alesin@bmstu.ru
} 


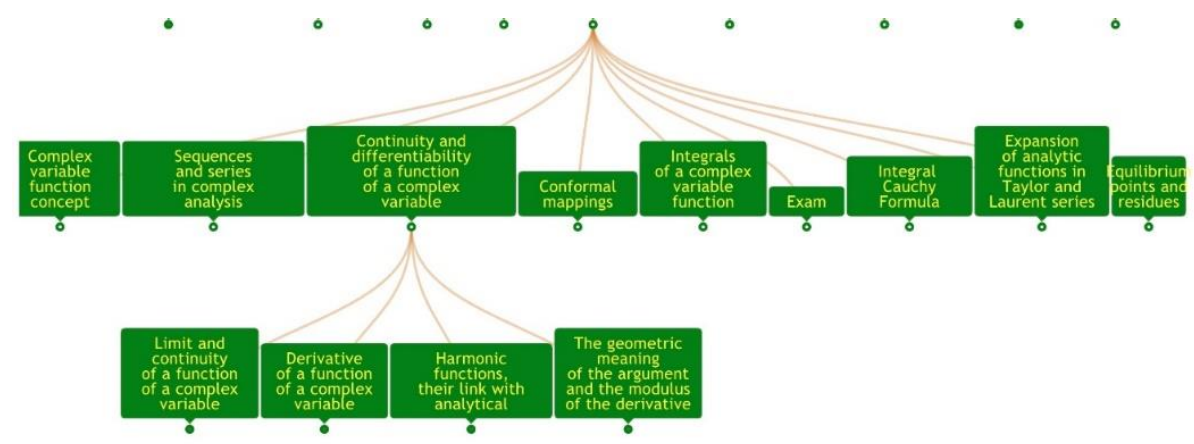

Fig. 1. The Chapters and paragraphs e-learning «Complex analysis» DLS NOMOTEX.

The practical sessions were developed according to the traditional curriculum and calendar of seminars, but supplemented with lots of materials that, before being developed, simply could not be into the curriculum. The introduction of the practical sessions were coincided with the unexpected introduction of distance learning in connection with of the pandemic in March 2020. During the transition to the new system of work, many teachers and students across the country were not ready for the difficulties that arose literally overnight. There was a lack of means of education: video conferencing systems and webinars were not available or were not able to provide simultaneous participation of a large number of participants.

Many, mainly age teachers, was simply confused. The course «Theory of functions of a complex variable» is the most important for students of faculties «Power Engineering», because its methods are widely applied in learning of various engineering disciplines. The department faced a serious task - to prevent disruption of the educational process, and it was solved with the help of DLS NOMOTEX. On the subject, teachers and students were offered a structured system of knowledge, with a mechanism for monitoring and checking student work. There was a technical opportunity to conduct distance classes - it was enough to provide voice communication during the demonstration of DLS NOMOTEX materials, which in modern realities is quite a feasible task. Broadcast with sloppy handwriting on the screen and broadcast from the NOMOTEX Knowledge Base (DLS) simply cannot be compared. The course of seminars on the "Complex Analysis" module, like other courses, is supported by the visualization of materials - a team of programmers has developed not just illustrations for tasks, but "living pictures", when the image changes instantly when the parameters of a task are changed on the screen (Fig. 2, 3).

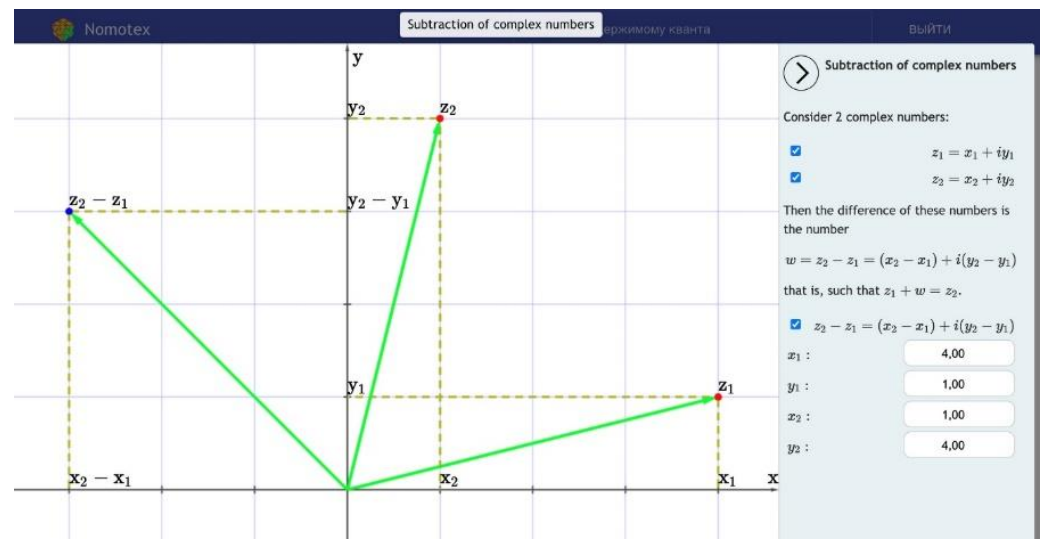

Fig. 2. Example of visualization in DLS NOMOTEX. 


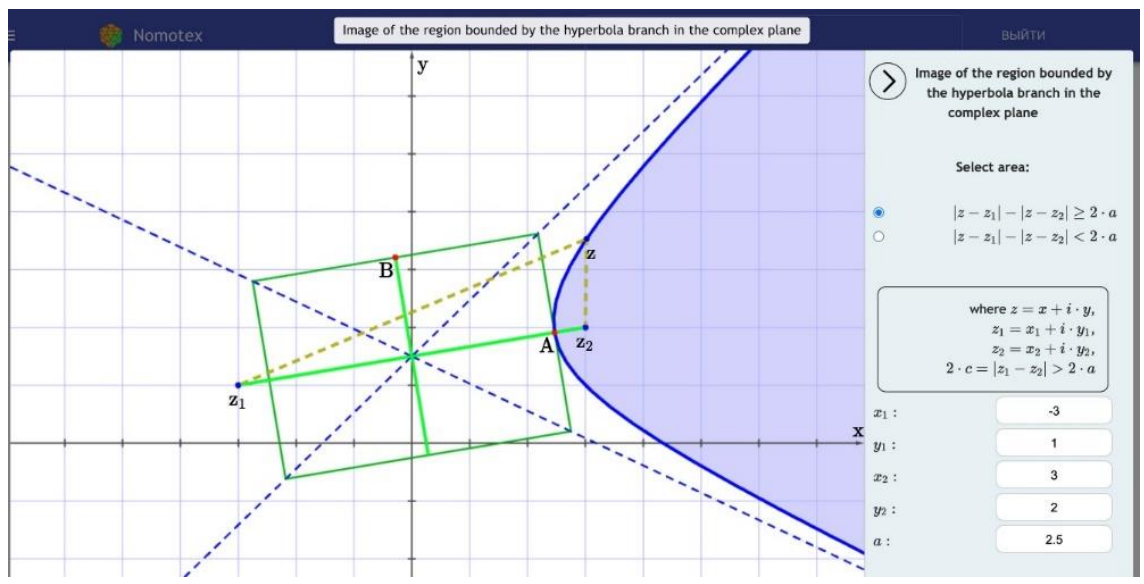

Fig. 3. Example of visualization in DLS NOMOTEX.

It is necessary to note the peculiarity of the Theory of Functions of a complex variable: the complex function maps areas, lines and points on the complex plane, onto areas, lines and points of the complex plane, while some functions of a complex variable are multivalued, others are multivalent. Such displays are first encountered by students, and understanding and awareness of what is happening is not easy for them. Therefore, the presence of visualization for this subject is especially important.

Visualization is not the only achievement of the team of programmers. The advantages of the proposed platform are in the unique developed software. The main distinguishing feature of DLS "Nomotex" from other educational programs is the verification of the answer entered by the student. Verification is multi-stage: what the student enters is transformed using a variety of mathematical functions until the answer is true. This allows the student to enter numbers and symbolic expressions in any form, since "Nomotex" does not require any additional actions from him to bring the answer to the form indicated in the system as correct. In tasks involving the introduction of several answers (areas, points, etc.), the order of input is not important, and the number of points that can be obtained for a task depends on the number of correctly found answers. For example, if a student was able to correctly identify two areas out of four, he will receive half of the possible points. Many tasks have individual automated check functions. Basically, these are those who do not have the only correct answer. In this case, the system itself makes the necessary transformations and concludes that the solution is correct. For example, if the task is to calculate an indefinite integral, the system will take the derivative of the entered answer and check it against the integrand. And, of course, he will not forget to additionally check the answer for the presence of a constant.

Initially, the DLS NOMOTEX tasks, regardless of the course, are built according to the principle of uniformity: each task has a number, is assigned to the corresponding section, and is titled in accordance with the topic. Available under the same number and title: "Seminar", "Homework", "Training test", "Test", "Exam" (Fig. 4).
18. Complex Analysis. Task 3b. Seminar
19. Complex Analysis. Task 4. Calculating the value of a function at a point. Homework
20. Complex Analysis. Task 4. Calculating the value of a function at a point. Test
21. Complex Analysis. Task 4. Calculating the value of a function at a point. Training test
22. Complex Analysis. Task 4. Calculating the value of a function at a point. Seminar
23. Complex Analysis. Task 4k. Test
24. Complex Analysis. Task 5. Test

Fig. 4. Example of tasks structure in DLS NOMOTEX. 
Thanks to this structuring, methodological connections of all events occurring during the study of the course are provided.

In the "Seminar" always at the beginning the text of the assignment is formulated and the Algorithm for solving the problem is presented. There must be brief theoretical information and necessary formulas. If necessary, active links to lecture material are added to the Algorithm. Since Nomotex is taught only when connected to the Internet, you can use the link immediately.

Also, in the "Seminar" several examples on this specific topic are necessarily presented, but selected so that when considering all the examples, you can understand all the nuances of the proposed topic.

Note that in the classical methodological manual, due to the limited publishing capabilities, it is extremely rare for several similar tasks to be present, especially in the Complex Analysis course - because of its volume. The tasks in DLS NOMOTEX are solved in detail, the choice of the solution method is justified, calculations are given - therefore, we can talk about the high methodological efficiency of teaching in this digital environment.

In "Self-study" tasks for training are presented, which are similar to tasks from the schedule for the subject. At the moment, the developers of Nomotex did not come to a consensus on the award of points for the performance of this work, since it is not individual for the student while the issue is under discussion.

In contrast to the "Independent work" in the "Homework" there is a typical individual task for each student who applied for it through the Personal Account. Currently, work is underway so that in all subjects the typical Homework is formed like the tasks of the "Test" - from a large library of tasks in a random way, which practically eliminates the scourge of classical teaching of the subject - the universal cheating of tasks from the stands, the same offered by the departments from year to year.

Since each task of the "Homework" is associated with the "Seminar" of the same name, the student immediately understands the methodology for completing each task. The computer checks the correctness of the answer, freeing up teaching time. Points are awarded for completing Homework. For homework and test work, the student is given points in accordance with the point-rating system adopted at the university [4]. To account for points in DLS NOMOTEX, a specialized subsystem has been created - the Personal Account, in which detailed information about each completed or not completed task (about each task) is recorded.

Tasks of the "Test work" in the current mode can only be seen by the teacher from his Personal Account, the student receives a randomly generated ticket from a large set of tasks. Thus, it is almost impossible to write off the test. The implementation of the Test work is always limited in time, which further mobilizes the student. Everything that a student performs remains in the computer's memory, and a teacher admitted to teaching in a group can always sort out his mistakes with the student and give recommendations.

The student enters the answers to the problems independently in the system with the ability to enter irrational numbers, functions of numbers, and so on. When solving homework, the student is given an unlimited number of attempts to enter the answer. The entered answer is instantly checked, and the student has the opportunity to immediately find out his result and double-check the solution, re-enter the answer if it was incorrect.

In the 2018-2019 academic year, an exam was held on the "Theory of the function of a complex variable" in the Digital Learning System NOMOTEX for students of the Faculty of Power Engineering, Bauman Moscow State Technical University. The exam ticket includes four tasks and a theory test. The student response to the DLS NOMOTEX study, including the midterm test and exam, was positive and the exam results were very satisfactory. 


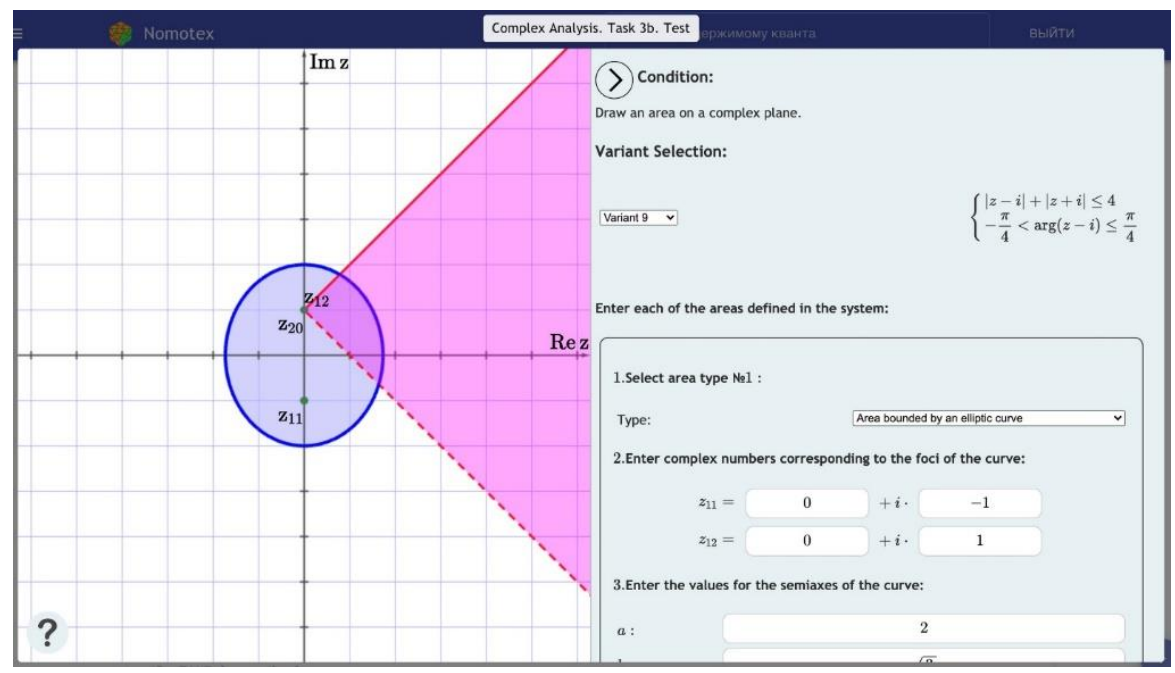

Fig. 5. Typical assignment for the course "Complex Analysis" in DLS NOMOTEX (from examination card).

The exam card implements such a complex task as the image on the complex plane of an area that satisfies the system of inequalities (Fig. 5). The student enters each of the areas specified in the system, and then selects the area that will be the intersection. All this is illustrated in parallel on the plane to the left of the problem statement.

In the future, the developers of DLS NOMOTEX set themselves an interesting task - so that the Nomotex points are exported to the Electronic University. This will facilitate the work of teachers, eliminate the corruption component.

Another important advantage of the DLS NOMOTEX environment is the presence of an interactive whiteboard screen. At any time, you can connect the Board mode, draw in different colors, write, calculate. Currently, using the interactive whiteboard and the DLS NOMOTEX environment, seminars are being recorded for students who find themselves on distance learning due to various circumstances. As a result, the listener sees on the screen the real process of solving problems, hears the voices of his comrades - a feeling of presence is obtained. At any time, DLS NOMOTEX slides are displayed on the screen, which allows you to see materials in high quality, and subsequently work through the tasks yourself.

You can include a slide with formulas, and solve on the blackboard with chalk. All assignments are printed, students see them in front of them - no time is wasted on reading the assignment, writing conditions on the blackboard, poring over an illustration, no need to carry a textbook with you - sometimes the weight of textbooks is quite large. All this saves time and effort for students and teachers.

The presence of the screen helps in considering cumbersome tasks that simply cannot be mastered during the seminar. Complex calculations can be demonstrated in the presentation mode, which gives students the opportunity to evaluate the finished result and, if desired, familiarize themselves in detail with all calculations at home. No methodical manual can afford such a volume of tasks. And DLS NOMOTEX can, which is another of its indisputable advantages.

At the moment, it was decided to supplement the course of complex analysis with applied problems solved at the engineering departments. To this end, the developers of DLS NOMOTEX turn to colleagues from the Faculty of Power Engineering to demonstrate how the materials studied at the Department of Mathematics in 1 and 2 courses are used in the study of engineering disciplines. 
In conclusion, we note that the base of tasks can be replenished and changed, which makes the Nomotex environment dynamic, lively, constantly changing, responding to the requirements of the time. One cannot say about it: "what is written with a pen cannot be cut out with an ax". Nomotex developers work in constant communication with teachers, immediately correct mistakes and inaccuracies, make the Nomotex environment convenient, comfortable and interesting for students and teachers.

\section{References}

1. Yu.I. Dimitrienko, E.A. Gubareva, New technology of mathematical training of engineering personnel, based on a neural network model of knowledge, Innovation in education, No. 11, pp. 129-140 (2017)

2. Yu.I. Dimitrienko, E.A. Gubareva, K.M. Zubarev, S.S. Kudryavtseva, Methodological support of the continuing education of teachers on the development of online courses at bmstu, Science diary, No. 11 (2018)

3. Yu.I. Dimitrienko, E.A. Gubareva, Hierarchical neural network model for mathematical knowledge and software NOMOTEX for mathematical training of engineers, IOP Journal of Physics: Conference Series, Vol. 1141012010 (2018). doi:10.1088/1742-6596/1141/1/012010

4. Yu.I. Dimitrienko, E.A. Gubareva, T.V. Oblakova, Methodology for assessing knowledge on the course "Mathematical Analysis" in the digital environment NOMOTEX, Innovative development, No. 9, pp. 8-11 (2018)

5. J. Engelbrecht \& A. Harding, Technologies involved in the teaching of undergraduate mathematics on the web, Journal of Online Mathematics and its Applications (2004)

6. J. Engelbrecht \& A. Harding, Teaching undergraduate mathematics on the internet, Part 2: Attributes and possibilities, Educational studies in mathematics, pp. 253-276 (2005)

7. B. Pepin, J. Choppin, K. Ruthven \& N. Sinclair, Digital curriculum resources in mathematics education: foundations for change, ZDM, 49(5), pp. 645-661 (2017)

8. A. Weinberg \& M. Thomas, Student learning and sense-making from video lectures, International Journal of Mathematical Education in Science and Technology, 49(6), pp. 922-943 (2018)

9. T. Jadin, A. Gruber \& B. Batinic, Learning with E-lectures: The meaning of learning strategies, Journal of Educational Technology \& Society, 12(3), pp. 282-288 (2009)

10. D. Pritchard, Where learning starts? A framework for thinking about lectures in university mathematics, International Journal of Mathematical Education in Science and Technology, 41(5), pp. 609-623 (2010)

11. C. Yoon \& J. Sneddon, Student perceptions of effective use of tablet PC recorded lectures in undergraduate mathematics courses, International Journal of Mathematical Education in Science and Technology, 42(4), pp. 425-445 (2011)

12. C. Yoon, G. Oates \& J. Sneddon, Undergraduate mathematics students' reasons for attending live lectures when recordings are available, International Journal of Mathematical Education in Science and Technology, 45(2), pp. 227-240 (2014)

13. M. Inglis, A. Palipana, S. Trenholm \& J. Ward, Individual differences in students' use of optional learning resources, Journal of Computer Assisted Learning, 27(6), pp. 490$502(2011)$ 
14. S. Trenholm, L. Alcock \& C.L. Robinson, Mathematics lecturing in the digital age, International Journal of Mathematical Education in Science and Technology, 43(6), pp. 703-716 (2012)

15. S. Trenholm, B. Hajek, C.L. Robinson, M. Chinnappan, A. Albrecht \& H. Ashman, Investigating undergraduate mathematics learners' cognitive engagement with recorded lecture videos, International Journal of Mathematical Education in Science and Technology, 50(1), pp. 3-24 (2019) 